\title{
Fabrication of self-assembled monolayer using carbon nanotubes conjugated 1-aminoundecanethiol on gold substrates
}

\author{
Mohammed M. Rahman
}

Laboratory of Interface and Surface Science, Department of Chemistry, Chonbuk National University, Jeonju, Republic of Korea; mmrahmanh@gmail.com

Received 7 December 2010; revised 26 January 2011; accepted 29 January 2011.

\begin{abstract}
The carbon nanotube (fundamentally Singlewalled carbon nanotube, SWCNT) based on 1-Amino-undecanethiol (AUT) were extremely controlled (nano-level) organizing a vertical self-assembled monolayer (SAM) on gold single crystal surfaces. The produced nano-surfaces were explored particularly by Fourier Transform Infra-red Spectroscopy (FT-IR), Cyclic Voltammetry (CV), Raman spectroscopy, Electrochemical quartz crystal microbalance (EQCM), and Atomic force microscopy (AFM) techniques. The SWCNTs were initially cut (chemically) into short pipes and thiol-derivatized at the open ends. The vertical aggregation of SWCNT-AUTs on chemically refined $A u(111)$ substrates was made-up by their spontaneous chemical bonding among carboxyl derivatized SWCNT-COOH and AUT SAM on Au(111), via peptide bonds, or directly by synthesized SWCNT-AUT composites. Raman spectroscopy and AFM surface images obviously disclosed that the SWCNTAUT (dia. 20 40 nm) has been vertically categorized d on gold (111) substrates, shaping a SAM with a perpendicular direction.
\end{abstract}

Keywords: SWCNT-AUT; Self-Assembled Monolayer; Surface Coverage; EQCM; Tapping-Mode AFM; Raman Spectroscopy

\section{INTRODUCTION}

Carbon nanotubes (CNTs) have appeared as an attracting new category of electronic materials owing to their nano-scale dimensions and exceptional properties, which comprise the capability to conduct a current density three orders of degree higher than distinctive conductors, such as copper and aluminium and the aptitude to conduct electrons statically. As unfilled cylindrical tubes prepared of whole carbon with enormously high aspect ratios (length/diameter), CNTs have one, two or few concentric graphite layers capped through fullerenic hemispheres. SWCNTs have been developed as an interesting candidate for fundamental studies since its possible applications including miniature chemical, biological, material as well as electronic devices. SWCNTs are one dimensional conductor or composer with all electrons moving in an atomic layer having surface atoms. The small dimensions, strength, and the extraordinary physical properties of these structures construct them an extremely exceptional material with a wide range of promising applications [1]. Since the innovation of carbon nanotubes by Iijima in 1991 by transmission electron microscopy, SWCNTs have been the topic of numerous investigations in chemical, physical, and material areas owing to their novel structural, mechanical, electronic, and chemical properties [2]. Depending on their atomic structure, SWCNTs act an electrically as a metal or as a semiconductor [3]. The functionalization of SWCNTs has concerned enormous interest in the past few years. These works are of large significance to survey the potential application for SWCNTs. The first work was conceded out by Green and co-workers [4] when they cut SWCNTs in short pipes using concentrated oxidizing agent. Self-assembly of a molecular monolayer onto a solid surface is an essential and technical way to build structurally controlled and stable organic thin films. Many applications of this stable, closely packed monolayer on solid substrates have been examined, counting adhesion, lubrication, promotion, corrosion inhibition, and microelectronics production. Several sorts of organic compound on solid substrates are utilized in SAMs. SWCNTs are two dimensional nanostructures with distinctive transport, electrical, electronic, and optical properties. The prospective use of SWCNT in electronic applications motivates research on their 
chemical properties and assemblies on the devices or electrodes. Pristine SWCNT materials are frequently collected of nanotubes of intently dispersed diameters, but their self-assembly layer more disseminated, random, mostly asymmetrical, and haphazard. SWCNTs can be functionalized with unusual chemical groups using covalent and non-covalent methods. Functionalized SWCNTs are then conjugated to diverse detection or recognition molecules for various potential applications.

Different objective analytes can be oxidized by SWCNTs at low potentials with minimum surface fouling, an appealing characteristic for the improvement of surface modification with high selectivity, stability, and reusability. Non-covalent functionalization of SWCNTs is important and fundamental to protect the $\mathrm{sp}^{2}$ nanotube structure and thus their significant electronic characteristics. Most of the inspected SAM systems use sulfur-anchor assemblies (thiol, di-sulfide, or thio-ether) and gold electrodes [5], since the chemical limpness of the substrate and its sturdy communication with sulfur permit a simple preparation of well-defined SAMs on single crystal gold surfaces. Because of strong intermolecular Vander Waals forces of interaction, such SAMs are compactly crammed and extremely vertical prearranged [6]. Significant development along these lines was lately attained by Liu and co-workers [7]. In this previous exertion, long SWCNT ropes were cut into short lengths of pen-ended and chemically functionalized tubes by oxidation in concentrated sulfuric and nitric acids [8]. Herein, it is reported the primary chemical congregation of SWCNT fabricated by the wet SAM techniques. Hence to offer common means of self assembly, we herein confirmed the perpendicular thiolfunctionalized SWCNT-AUT monolayers on gold single crystal surfaces via peptides chemical bonding directly or indirectly. For most of these applications, well-ordered nanotubes with aligned orientations are greatly enviable, but conventional methods [9] only formed carbon nanotubes in an arbitrarily tangled situation. Consequently, there is a vast confront to unwrap up a new technique to categorize the SWCNTs into well-ordered arrays. Based on SAMs of molecular chains, we put frontward a innovative approach to assemble two-dimensional arrays of SWCNT by a chemical approach for the development of electrodes or electronic devices.

\section{EXPERIMENTAL METHODS}

\subsection{Reagents and Materials}

Potassium ferricyanide (III) (99\%) and Dicyclohexylcarbodimide (99\%) are obtained from Aldrich Chemical Company. Sulphuric acid (97\%), nitric acid (60\%), Tri- ton X-100 (98\%), and hydrogen peroxide (30\%) are purchased from Showa Chemical Company (Japan). All the reagents are in analytical grade, used as received without additional purification. Solutions are prepared in deionized distilled water (resistivity, >18.2 M $2 . \mathrm{cm}$ ). The commercial existing SWCNTs dispersed solution and 1-Amino-undecanethiol (AUT, $\mathrm{NH}_{2}\left(\mathrm{CH}_{2}\right)_{11} \mathrm{SH}$ ) is used to derivatize the SWCNT-COOH and SWCNT-AUT SAM, which is purchased from Tubes@Rice Company.

\subsection{Fabrication of SAM on Quartz Crystal Electrode}

All quartz glasses were originally cleaned by piranha solution (a mixture of $98 \% \mathrm{H}_{2} \mathrm{SO}_{4}$ and $30 \% \mathrm{H}_{2} \mathrm{O}_{2}$ at $2: 1$ $\mathrm{v} / \mathrm{v}$ ) for ca. 10 minute [Caution! Piranha solution is a extremely strong oxidizing agent and reacts aggressively with organic compounds. Storing in a closed container and revelation to direct contact should be evaded. Freshly arranged piranha solution should be handled with excessive concern]. It was then rinsed with deionized water and ethanol and then dried with a stream of $\mathrm{N}_{2}$ gas. The cleaned gold coated quartz electrode is immersed in ethanolic solution of AUT and SWCNT-AUT. Then the modified electrode systematically washed with ethanol and dried with nitrogen gas. All electrochemical measurements were conceded at room temperature after the electrolyte solution was de-aerated by purging $\mathrm{Ni}$ trogen gas for at least 10 minute.

\subsection{Electrochemical Technique}

Cyclic voltammetry experiments were performed using an electrochemical analyzer (Shin1000, Electrochemical Quartz Crystal Microbalance, EQCM, Korea) with a conventional three-electrode cell. All of the voltammetric measurements were carried out in a one compartment cell having the naked or AUT-SAM, SWCNTAUT SAM modified gold as a working electrode, a Pt wire as a counter electrode and a $\mathrm{Ag} \mid \mathrm{AgCl}$ (saturated $\mathrm{KCl}$ ) as a reference electrode. The EQCM was executed using frequency counter (Shin Cor. Model EQCN1000) with potentiostat and gold coated AT cut quartz crystal oscillator electrodes supplied by International Crystal Manufacturer. The resonance frequency of the oscillator was $10.0 \mathrm{MHz}$ and the frequency change of $1.0 \mathrm{~Hz}$ corresponds to $4.42 \mathrm{ng} / \mathrm{cm}^{2}$. The perceptible surface area of gold electrodes was $0.256 \mathrm{~cm}^{2}$ and roughness factor was about 1.2. Electrochemical reductive desorption of AUT and SWCNT-AUT SAM was employed in $0.5 \mathrm{M} \mathrm{KOH}$ aqueous solution. The solution in the cell was de-aerated by bubbling $\mathrm{N}_{2}$ gas for at least 30 minutes before performing the each electrochemical exploration. 


\subsection{Preparation and Purification of SWCNTs}

The SWCNTs are high quality and quite pure, although some nanoparticles still exist in the obtained material as a by-product. SWCNTs are produced either as isolated units or as nanotubes prearranged in bundles; no effort was made to separate the different patterns. The purification of general SWCNTs is of huge significance since most carbon nanotube applications require materials of high quality. Nitric acid is a familiar reagent for refinement of carbon nanotubes and has comprised the initial step in different purification methods. Nitric acid treatment is typically developed to eliminate metal catalysts, together with some of the amorphous carbon, but it can also oxidize carbon atoms at the ends [11]. Sonicating SWCNTs in nitric acid opens the ends of the nanotubes [12] and thereby commences carboxylic acid groups at the ends or defect sites of SWCNTs [13]. The preparation of SWCNTs functionalized with carboxylic acid groups was performed as follows. Firstly, SWCNTs were soaked in $5.0 \mathrm{M}$ nitric acid, and then ultrasonically dispersed them for 6 minutes. Secondly, these are diluted with a great quantity of water and add a little Triton X-100 surfactant to amplify the solubility, then sonicated it to be a black solution. Thirdly, the black solution was filtered with $0.2 \mu \mathrm{m}$ diameter film and composed the SWCNTs and then repeated the second and the third steps.

\subsection{Preparation of Gold Single Crystal Surfaces}

The single-crystal gold-coated glass plates were prepared according to Arrandee [10], by flame annealing, $\mathrm{Au}(111)$ terraces can be attained. Best is to flame annealing in a dark room, to be capable to examine the glowing of the gold arrandee by a propane/butane flame. Hydrogen flame was too high temperature. Gold arrandee was put in flame and taken out after heating and allocates cooling down for about 30 seconds. This process was repeated three times, then it was executed to locate $\mathrm{Au}(111)$ terrace of about $500 \mathrm{~nm}$ in diameter estranged by irregular boundaries.

\subsection{Instrumentations}

The measurements were taken at room temperature. IR spectra were measured by a FT-IR spectrometer (AVATAR 330, Thermo Nicolet. USA) as a $\mathrm{KBr}$ disc. Non-contact AFM mode (Solver NT-MDT, Nano-Finder, Japan) is used to confirm the SWCNT-AUT modified gold single crystal surfaces. Raman spectra (Raman Spectroscopy, KBSI, Japan) is obtained for SWCNTAUT samples concurrently throughout AFM measure- ment.

\subsection{Preparation of SWCNT-AUT SAM on $\mathrm{Au}(111)$}

SWCNT-AUT self-assembled is fabricated in two different approaches on gold substrates.

\subsubsection{One-Step Method for SWCNT-AUT SAM}

The SWCNT-AUT SAM was produced by plunging cleaned gold(111) electrode in the ethanol solution having $1.0 \mathrm{mM}$ of SWCNT-AUT for 48 hours. Subsequently it was systematically rinsed with ethanol and dried with $\mathrm{N}_{2}$ gas. The fabricated SWCNT-AUT gold(111) surface is considered by non-contact AFM images. The elevated compactness of SWCNT-AUT aggregated monolayers is employed. The condensation among the carboxylic termini of the SWCNTs and the amino group of the thiols (peptide bond) is demonstrated by the manifestation of an amide band approximately $1600 \mathrm{~cm}^{-1}$ in the FT-IR spectrum.

\subsubsection{Two-Step Method for SWCNT-AUT SAM}

The AUT SAM was produced by immersing a cleaned gold (111) electrode in the ethanol solution containing $1.0 \mathrm{mM}$ AUT for two hours. Subsequently it was comprehensively rinsed with ethanol and dried with nitrogen gas. The AUT SAM-modified electrode was immersed for 24-72 hours in activated solution of DCC along with SWCNT-COOH. Occasionally the activated solution was put in a refrigerator at about $4.0^{\circ} \mathrm{C}$ for above a week for adequate activation. It was afterward rinsed with ethanol and dried with nitrogen. Covalent attachment of SWCNT-COOH to the AUT SAM was induced by a coupling agent, which is DCC solution in ethanol. This procedure is commonly applied for configuration of amide bonds among amino groups and carboxylic functional groups in the peptide synthesis.

\section{RESULTS AND DISCUSSIONS}

Figure 1 exemplifies the essential schematic methodology of to immobilize the SWCNTs on gold via the surface condensation reaction in existence of condensation agent. The single crystal gold substrate is first modified with AUT self-assembled monolayer completed by amino groups. The AUT molecules were established to form compactly packed monolayers on gold as confirmation by electrochemical extents and high-resolution scanning tunneling microscopy images. The chemically shortened SWCNTs having carboxyl groups of AUT SAM on gold with the help of dicyclohexylcarbodiimide condensation agent at $4.0^{\circ} \mathrm{C}$. The condensation reaction among carboxyl and amino groups is well recognized 

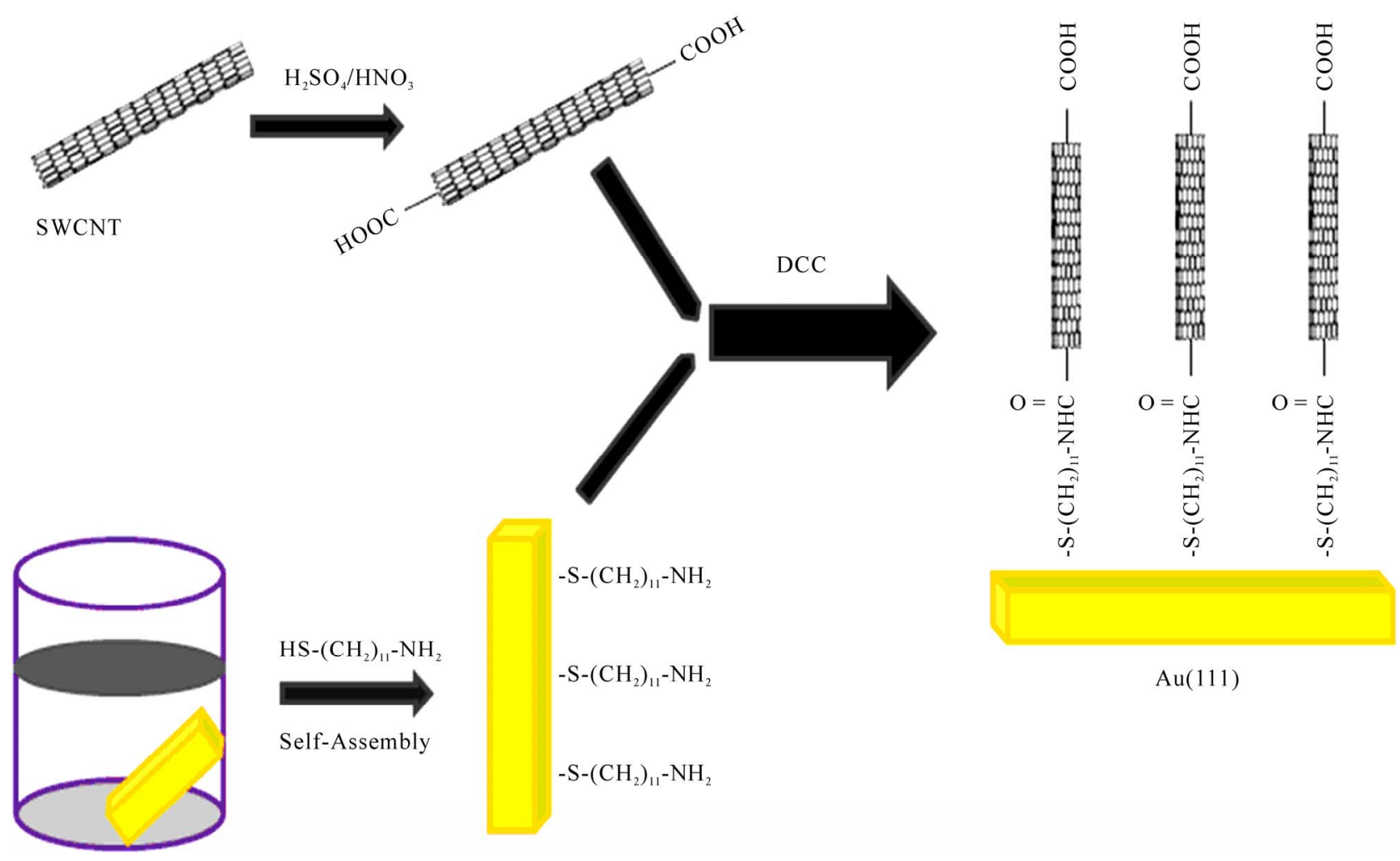

Figure 1. Schematic illustration of the surface condensation reaction (peptide conjugation) method for fabricating highly aligned SWCNT-AUT SAM on Au(111).

and often used in polypeptide synthesis. We suppose to immobilize the short SWCNTs on solid gold substrates in a extremely ordered manner using this attitude.

The carboxylic acid groups exhibit an essential function in SWCNT chemistry as they can be further reacted with a multiplicity of organic molecules [7]. In addition, to take away the metal catalyst commencing SWCNT, it is obtained that nitric acid demolishes SWCNT to generate carbonaceous impurities. The exclusion of the acidified amorphous carbon can be obtained via centrifugation [14,15]; though, the mechanism of this route and the function played by centrifugation has not been thoroughly premeditated. Liu et al. approach [16], the purified SWCNTs were cut into short pipes by chemical oxidation in a mixture of concentrated $\mathrm{H}_{2} \mathrm{SO}_{4}$ and $\mathrm{HNO}_{3}$ (3:1, 98\% and $70 \%$, respectively) under ultrasonication for 8 hours [17-19]. The same techniques were employed to carboxyl derivatized of obtained SWCNTs sample. The reaction mixture of SWCNTs was then diluted with water and permitted to set overnight for precipitation. The supernant was decanted, and the rest were diluted with deionized water and filtered with a $1.0 \mu \mathrm{m}$ (diameter) pore polytetrafluoroethylene membrane (PTFE, Gelman, commercial name) under vacuum. The solid shortened SWCNT sample was attained by washing the remains on the PTFE filter with deionized water until the filtrate $\mathrm{pH}$ became practically neutral. The SWCNT pipes attained were originated to form stable colloidal suspensions in water, ethanol, acetone, and dimetylformaldehyde (DMF). Suspensions were equipped by ultrasonication without using surfactants, suggesting that comparatively short nanotubes have been made as compared with Liu's work [7]. The characteristic stretching band $(\mathrm{vC}=\mathrm{O})$ of carboxylic groups at $1710 \mathrm{~cm}^{-1}$ in the FT-IR spectrum powerfully suggests the development of open-ended and carboxyl-terminated SWCNTs after oxidation treatment $[20,21]$.

\subsection{Cyclic Voltammetry}

Conventional electrochemical method, cyclic voltammetry $(\mathrm{CV})$ is the most versatile electroanalytical procedure for the investigation of electroactive species, and is extensively used in industrial applications and educational or technical research entities. CV is also an significant method to assess the blocking property of the monolayer-coated electrodes via diffusion controlled redox couples. Figure 2 exhibits the cyclic voltammograms of bare Au and SWCNT-AUT SAM-modified Au electrodes in $1.0 \mathrm{mM} \mathrm{K}_{3} \mathrm{Fe}(\mathrm{CN})_{6}$ with $0.1 \mathrm{M} \mathrm{KOH}$ as the supporting electrolyte at a potential scan rate at 100.0 $\mathrm{mV} / \mathrm{s}$. It can be seen from the Figure 2, that the nake 


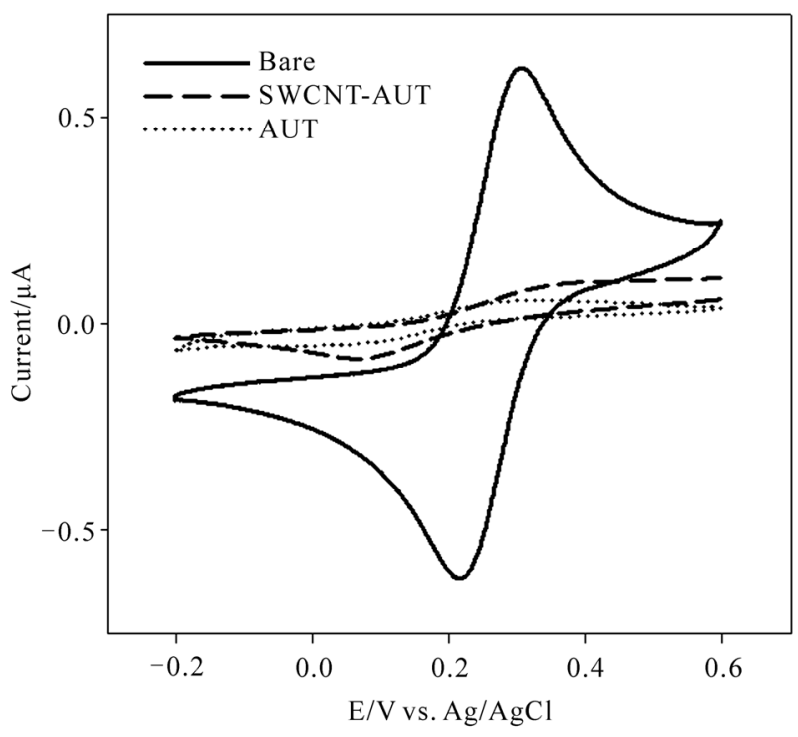

Figure 2. Cyclic voltammogram in $1.0 \mathrm{mM}$ potassium ferrocyanide with $0.1 \mathrm{M} \mathrm{KOH}$ as supporting electrolyte at a potential scan rate at $100 \mathrm{mV} / \mathrm{s}$ for bare $\mathrm{Au}$ electrode (solid line), dotted lines are SAM of SWCNT-AUT and AUT on Au surface, respectively.

gold electrode (solid line) exhibits a reversible voltammogram for the redox couple specifying that the electron transfer reaction is totally diffusion controlled. In disparity, the absence of any peak formation in the CVs of the SWCNT-AUT monolayer-modified electrodes presents that the redox reaction is slightly inhibited or blocked. It can also be perceived that in the case of AUT SAM (Figure 2), the CV shows an ideal blocking manner. So the CVs for SWCNT-AUT \& AUT designate a good blocking behavior for the electron transfer reaction, which denotes that a extremely ordered, condensed monolayer is produced on the Au surface [22].

\subsection{EQCM Study}

The surface coverage's of SWCNT-AUT (with considering roughness factor, 1.2) SAM on Au substrates is considered according the Eq.1. The charge (Q) recorded in bulk electrolysis experiments or the areas beneath slow scan rate cyclic voltammograms can be used in combination with lower equation to conclude the surface coverage $\Gamma$ of electroactive redox centers:

$$
\Gamma=Q / n F A
$$

where, $F$ is Faraday's constant and $A$ is the area of the electrode.

The desorption peak and mass change are calculated in $0.5 \mathrm{M} \mathrm{KOH}$ electrolyte at $25 \mathrm{mV} / \mathrm{s}$ scan rates. The desorption conduct of the thiol derivatized SWCNTAUT and AUT SAM was checked by EQCM method. After the SWCNT-AUT was accumulated on the Au, the surface coverage of the SWCNT-AUT compound was anticipated by reductive desorption. The abrupt amplified in frequency and cathodic current of AUT samples was found at around $-1.06 \mathrm{~V}$, which was accredited to the reductive desorption of the SAMs from the gold surface (Figure 3(a)). But for SWCNT-AUT SAM, current and frequency tainted in two steps during reductive desorption, first for SWCNT-AUT (at elevated potential) and second one for AUT (at lower potential, non bonded with SWCNT-COOH) desorption, which is shown in the Figure 3(b).

The CV's in the existence of AUT SAM exhibited desorption peak at $-1.06 \mathrm{~V}$ and surface full-coverage $4.61 \times 10^{-10} \mathrm{~mole} / \mathrm{cm}^{2}$ in the potential range 0 to $-1.3 \mathrm{~V}$ (Figure 3(a)). Adsorbate coverage is expected by integrating the charge under the voltammetric wave for the adsorbed complex, which shows two reductive desorption peaks like binary SAM at about $-1.08 \mathrm{~V}$ (for AUT) and $-0.51 \mathrm{~V}$ (for SWCNT-AUT) vs. $\mathrm{Ag} \mid \mathrm{AgCl}$ (saturated $\mathrm{KCl}$ ) at the scan rate $25.0 \mathrm{mV} / \mathrm{s}$ (Figure 3(b)). The experimental values of surface coverage's for AUT and SWCNT-AUT are intended individually $1.73 \times 10^{-10}$ and $2.71 \times 10^{-10} \mathrm{~mole} / \mathrm{cm}^{2}$ correspondingly. The desorption potential of AUT peak coincide with previous value at $-1.08 \mathrm{~V}$. The new peak at higher desorption potential region is executed for SWCNT-AUT self-assembled monolayer.

The calculated total surface coverage for total AUT (Figure 3(a)) full-coverage $\left(4.61 \times 10^{-10} \mathrm{~mole} / \mathrm{cm}^{2}\right)$ was incredibly similar to that for total desorption (Figure 3(b)) during SWCNT-AUT and AUT SAM (AUT $1.73 \times$ $10^{-10}+$ SWCNT-AUT $\left.2.71 \times 10^{-10} \mathrm{~mole} / \mathrm{cm}^{2}\right) 4.44 \times$ $10^{-10} \mathrm{~mole} / \mathrm{cm}^{2}$ within experimental error boundary. This signified that the monolayer produced via SWCNT-AUT had similar surface coverage to that of the AUT for extended immersion in the solution [23].

\subsection{Tapping AFM Images}

The chemical accumulated process offers an additional valuable, efficient, and suitable technique to construct SWCNT investigates for scanning probe microscopy studies [24-26], well proscribed aligning of nanotubes is of meticulous significance for generating nanotube-based nano-electronic and nano-molecular electronic devices or chips [27]. We have achieved in accumulating carboxylic acid derivatized SWCNTs on an amino-terminated $\mathrm{Au}(111)$ surface via electrostatic interactions. Nanotube assemblies offer broad potential for nanotube applications in electronic and optoelectronic devices. We consider that the occupied standing aggregation nanotubes on an electrode surface will significantly advanced the field-emission performances of electrons for flat-panel display applications [28], escalating 


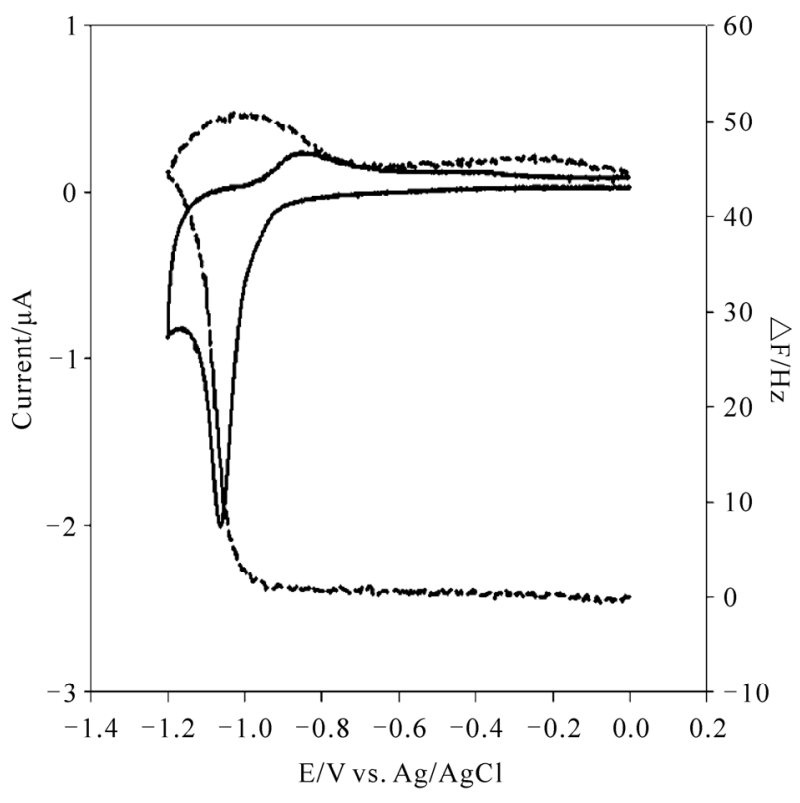

(a)

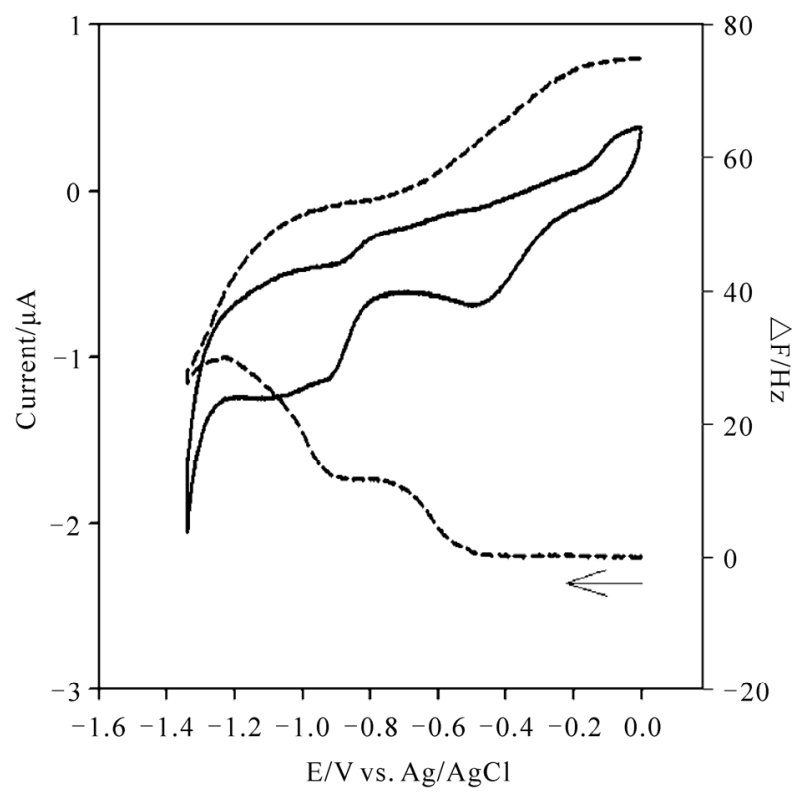

(b)

Figure 3. Desorption peaks of (a) AUT SAM and (b) SWCNTAUT SAM (direct sample's) in $0.5 \mathrm{M} \mathrm{KOH}$ at $25 \mathrm{mV} / \mathrm{s}$ scan rate, immersion time $24 \mathrm{~h}$.

the current density, lowering the operation voltage, and miniaturizing the device dimensions. It has been studied non-contact tapping mode atomic force microscopy (AFM) to exemplify the topograph of the thiol-derivatized carbon nanotubes on gold substrates.

Atomic force microscopy was employed to attain the structural information of the SWCNT assembly prepared on gold by the surface condensation procedure. Figure 4 represents the distinctive aggregation of SWCNT-AUT
AFM images acquired, where (A) two-dimensional (2D) and height profiles correspond to the lines drawn in the 2D image, (B) three-dimensional (3D), and (C) statistical distribution in SWCNT-AUT diameter. Figure 4 is an tapping mode AFM two and three dimensional representations of an attached SWCNT-AUT self-assembled monolayer on $\mathrm{Au}(111)$ surface. Each bright-spot (twodimensional) or standing (three-dimensional) characteristic is accredited to an adsorbed SWCNT-AUT SAMs. Unlike the predictable organic self assembling molecules on solid surfaces (e.g., alkanethiols on gold), the carbon nanotubes immobilized on gold have dissimilar lengths and form various-sized of aggregates. This examination is extremely reproducible at different sites of SWCNT through oxidation of SWCNT samples. The diameter of the SWCNTs unswervingly computed from tapping mode AFM images falls in the range of ca. 20-40 nm. The large diameter resulting from the AFM image may be owing to the tip broadening consequence. When the sample is high and steep, the contour line reported by the AFM can be relatively unusual from the accurate contour of the sample. In the tremendous case, where the sample is very high and sharp contrast with the tip, the AFM image is an image of the tip rather than the sample. Under this condition, the SWCNT-AUT with sharp geometrical pattern acts as an AFM tip to image the large innovative AFM tip. The accurate image can be reconstructed by deconvolution. For the nominal lateral size examined from the AFM image, the deconvolution presents a true tube diameter of ca. $20-40 \mathrm{~nm}$.

Aggregations are apprehended by two or more SWCNT-AUTs simultaneously in dissimilar lengths on gold surface, its may be owing to the hydrogen bonding among the neighbor carboxyl-derivatized SWCNT walls [29] after chemical treatment. The widths of SAM geometry are considered; the aggregated diameter is broadening by double, triple or more SWCNT-AUT tubes on gold surface. The largest diameter of nanotubes from AFM data (ca. $30 \mathrm{~nm}$ ) gives a cumulatived diameter of ca. $120 \mathrm{~nm}$. The innovative chemically condensed SWNT suspension controlled different lengths of nanotubes up to $200 \mathrm{~nm}$, which is investigated by AFM results. Consequently, the above AFM data specify that only comparatively short tubes can be immobilized on gold surfaces via a condensation reaction. A related conclusion has been drawn Liu et al. [7], in which the thiolated nanotubes were deposited or adsorbed on gold via Au-S chemical bonds. On the other hand, the experimental lateral dimensions of SWCNT descend into a range of 20 to $120 \mathrm{~nm}$, generally within $20-45 \mathrm{~nm}$ (82.1\%). Because of the broadening effect of the AFM tip [28], these data do not straightforwardly reflect the true lateral sizes of nanotubes. Most of the SWCNTs in 


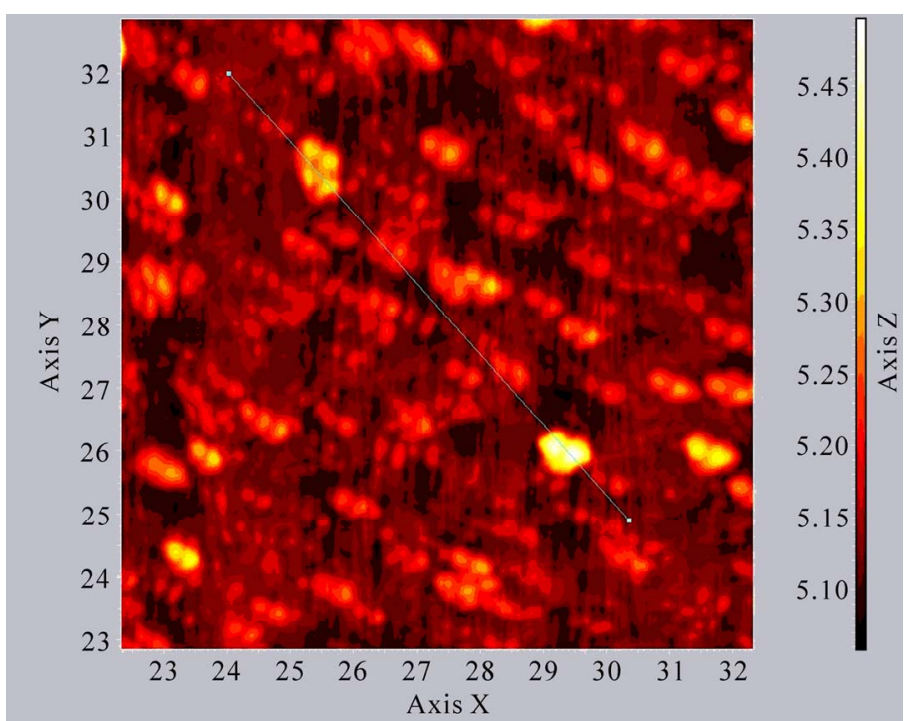

(a)

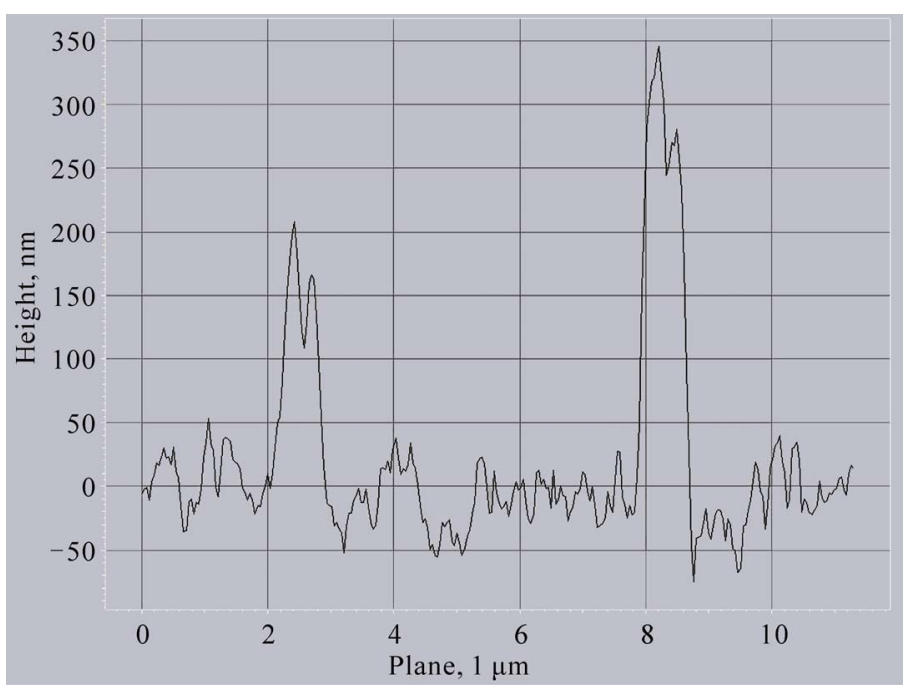

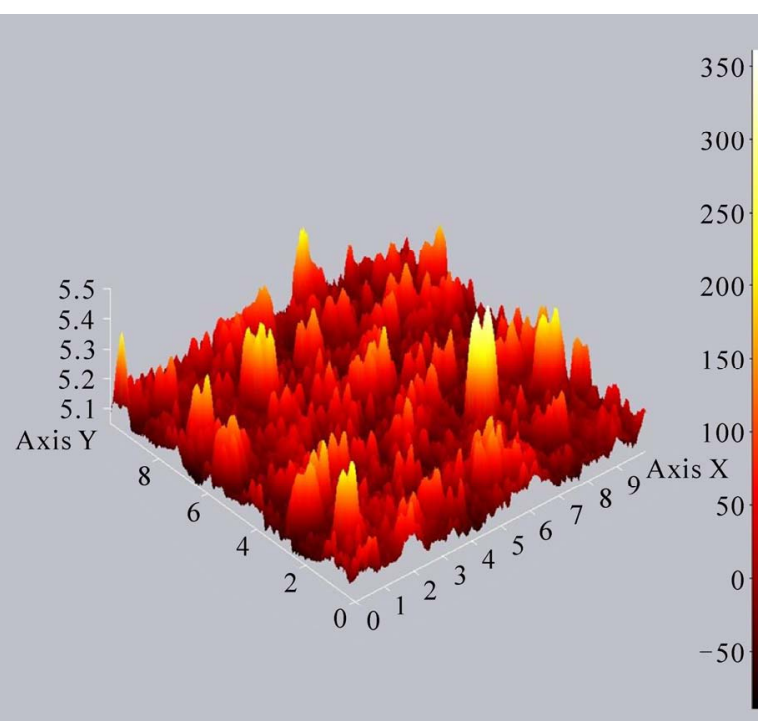

(b)

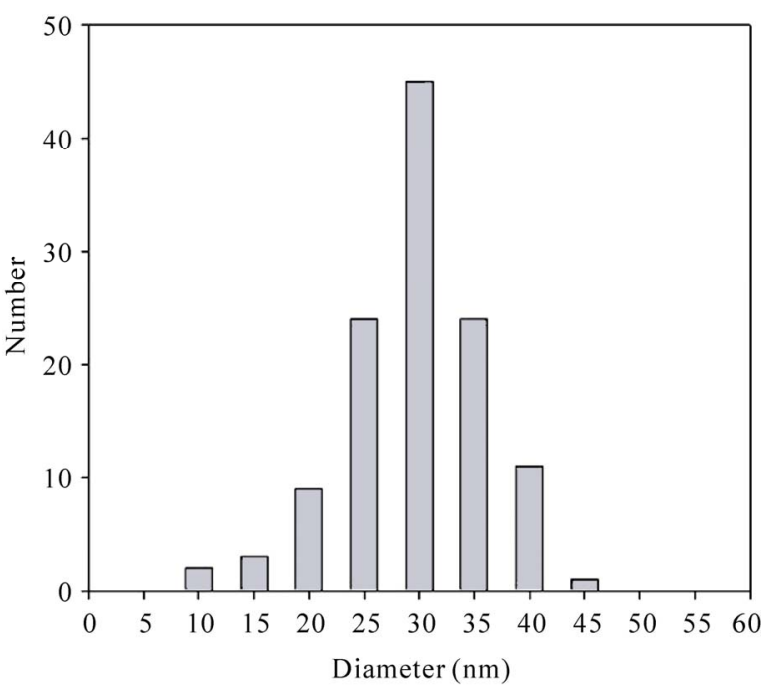

(c)

Figure 4. AFM images $(1000 \mathrm{~nm} \times 1000 \mathrm{~nm})$ of SWCNT-AUT SAM (AUT SAM is immersed in ethanolic SWCNT-COOH solution) on $\mathrm{Au}(111)$ substrates, Immersion time 24 hours, (a) 2D-image \& height profiles correspond to the lines drawn in the 2D AFM image, (b) 3D-image, (c) Statistical distribution in SWCNT-AUT diameter.

the assembly subsist in aggregated shapes, representing that the carbon nanotubes tend to form aggregates, that is, bundles during surface condensation. Approximately all bundles probable have fine saw-tooth structures, signifying the subsistence of dissimilar lengths of nanotubes within individual bundles. The carbon nanotubes are carboxylated at both open ends. Both carboxylic termini may have the same prospect of contributing in the surface condensation reaction. However we never found nanotubes lying flatly on the surface. In fact, the fine saw-tooth structures found in the cross section also propose the standing-up orientation because otherwise we would observe a flat cross section for the stacked nano- tubes along the long axis direction. This is logical because direct contact among the hydrophobic nanotube side walls and the hydrophilic amino surface is actively inauspicious. It should be pointed out that the nanotubes may tilt some degree from the surface normal, depending on how many carboxylic groups at the tube end (typically not one) have participated in the condensation reaction and on the inter-nanotube interactions. The cutting angle of the nanotube at the open end formed during the oxidative shortening process may also influence its tilt angle on the surface.

The present studies demonstrate that aggregates SWCNT-AUT SAM can be chemically assembled on gold 
surfaces using a similar AUT or some other alkanethiols as amine terminal organic self-assembled species. The SAM was organized through the covalent attachment of carboxylic derivatized single-walled carbon nanotubes (SWCNT-COOH) in an activation solution having DCC coupling agent to AUT SAMs produced on a gold substrate in two ways at same immersion time interval (Figure 5). AFM images of SWCNT-AUT were executed in two ways. Direct SWCNT-AUT SAM is employed from the ethanolic solution of SWCNT-AUT sample in single step method. The elevated density of SWCNT-AUT aggregated monolayers is formed (Figure 5(a)). The condensation among the carboxylic termini of the SWCNTs and the amino group of the thiols (peptide bond) is evidenced by the appearance of an amide bond about $1600 \mathrm{~cm}^{-1}$ in the FT-IR spectrum. In two steps method, covalent attachment of SWCNT-COOH on the AUT SAM (AUT SAM made before immersion in SWCNT-COOH ethnolic solution) was induced by a coupling agent (DCC) in ethanol. This method is extensively applied for formation of amide bonds between amino groups and carboxylic functional groups in the peptide synthesis. Less number of SWCNT-AUTs SAM is formed, which is presented in Figure 5(b). This is may be owing to the DCC covered carboxylic derivatized SWCNT walls, so less activity to make aggregation and bond formation with AUT SAM. The DCC-aided condensation reaction between carboxyl and amino group proceeds in two successive steps: DCC molecules react first with the carboxyl group, generating an active ester intermediate, which then reacts with the amino species. In the present system, DCC is excessive in magnitude contrasted with the SWCNTs, while the amino groups are bound to solid substrates. Since the ester intermediates are quite stable in ethanol, we supposed most of the nanotubes exist in the intermediate form, and the effective collision of the intermediates to the amino-terminating surface controls the condensation process. The oxidatively shortened nanotube typically has more than one carboxyl group at one end. These carboxyl groups may combine more than one DCC molecule, generating an ester intermediate with multiple active sites. This would facilitate the surface pinning of huge nanotubes. It should be noted that the outermost carboxyl groups of the immobilized SWCNTs on gold may also be coupled with DCC after the condensation reaction. However, these ester intermediates will be instantly hydrolyzed to the innovative carboxyl groups after rinsing in water. As noted above, the carbon nanotubes tend to form bundles during the collision process. This may be accredited to the strong attractive interactions between the hydrophobic sidewalls of SWCNTs. It also suggests that the collision may not directly lead to the condensa-

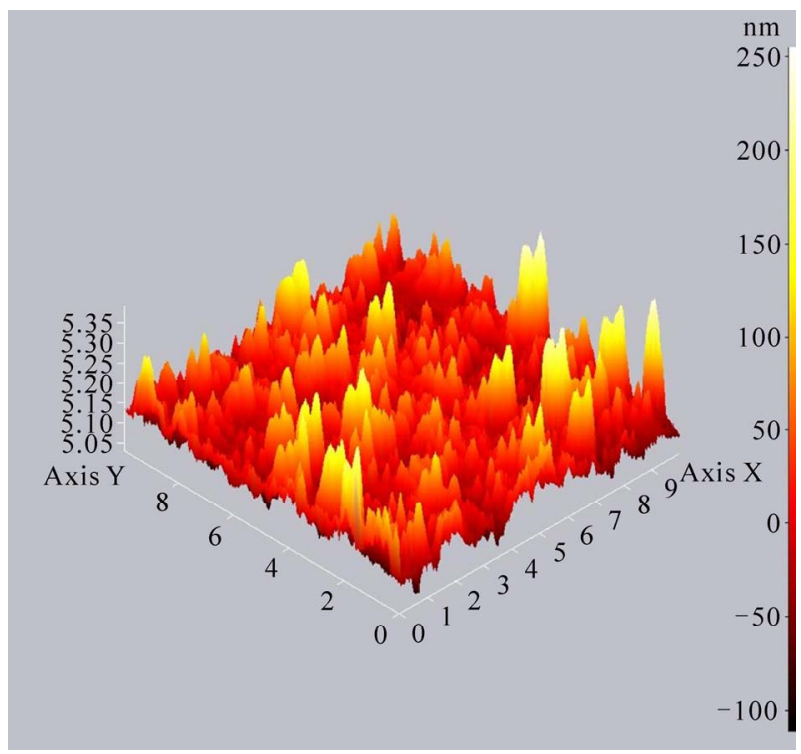

(a)

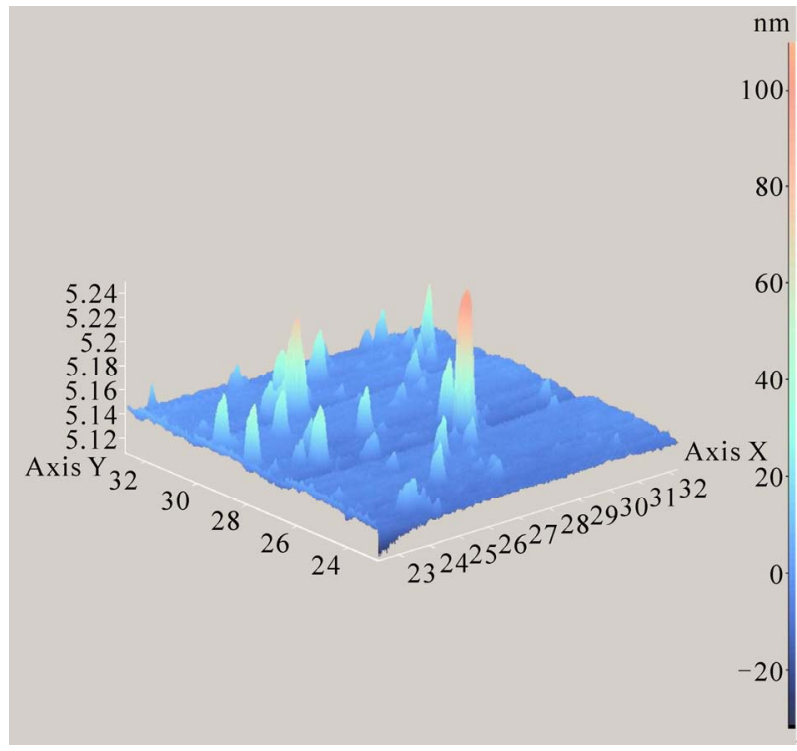

(b)

Figure 5. AFM images of SWCNT-AUT (a) direct SWCNTAUT sample's (b) via peptide bond (AUT SAM immersed in ethanolic solution of SWCNT-COOH) on $\mathrm{Au}(111)$ substrates. Immersion time 36 hours, non-contact AFM mode, and $1000 \times$ $1000 \mathrm{~nm}$.

tive pinning of nanotubes, and the following lateral movement of the intermediates on surface would be possible. At the initial stage, the collision of nanotube-carrying ester intermediates with the surface amino groups consequences in reactive immobilization of SWCNT on gold at few locations. These immobilized individual nanotubes may have different diameters and serve as nucleation centers. The following nanotubes then preferentially adsorb surrounding these nuclei, leading to the 
formation of various nanotube self-assembly.

\subsection{Raman Spectroscopy}

Raman spectroscopy is one of the most influential methods for classification and characterization of nanomaterials, semiconductor materials, and carbon nanotubes etc. All allotropic forms of carbon like fullerenes, carbon nanotubes, amorphous carbon, polycrystalline carbon, etc. are active in Raman spectroscopy [31]. The position, width, and relative intensity of bands are modified according to the carbon forms [32]. This procedure provides valuable information concerning the structure of carbon nanotubes. Shortly, there is strong substantiation for a diameter-selective resonant Raman scattering process. The tangential mode (TM) in the range 1400$1700 \mathrm{~cm}^{-1}$ gives information on the electronic properties of the tubes while the analysis of the so called D-band at around $1360 \mathrm{~cm}^{-1}$ provides information as to the level of disordered carbon. The size of the D-band relative to the $\mathrm{TM}$ band is a qualitative computed of the formation of unattractive forms of carbon. In this experiment, it is utilized 788-nm (semiconductor Sapphire Laser) excitation for checking SWCNTAUT self-assembled monolayer on gold single crystal substrates. This is the most direct confirmation of SWCNT- AUT SAM on $\mathrm{Au}(111)$, which is directly detected by Raman spectroscopy. The Raman spectrum of the SWCNT-AUT modified gold surface shown in Figure 6, where the G-line at $1598 \mathrm{~cm}^{-1}$ originates from the graphitic sheets [33] and the peak at $1365 \mathrm{~cm}^{-1}$ is related to the defects (disorder mode consistent with sidewall functionalization) in CNTs [34]. From this, we can also conclude that the physical structure of the CNTs was not distorted with the only exception of the opened ends.

\section{CONCLUSIONS}

A chemical approach was place onward to categorize and organize the arbitrarily chemisorbed SWCNT-AUT SAMs on a $\mathrm{Au}(111)$ substrate. From all of these experimental scrutinies, it is confirmed that the thiol-derivatized SWCNT-AUT have been effectively immobilized on $\mathrm{Au}(111)$ via $\mathrm{Au}-\mathrm{S}$ chemical bonding, with the nanotubes being vertically aggregated and standing on the gold surfaces. We established that SAM of SWCNT- AUT is characterized and developed, and the adsorbed arrays will offer broad possibilities for different purposes. Using Raman and AFM, we demonstrated that extremely stable, vertically aligned, and patterned SWCNT-AUT assemblies on gold single crystal were executed. It was also investigated that the massive SWCNT-AUT tends to form bundles on amino-terminating surfaces. This type of extremely aligned nanotube assembly may afford wide

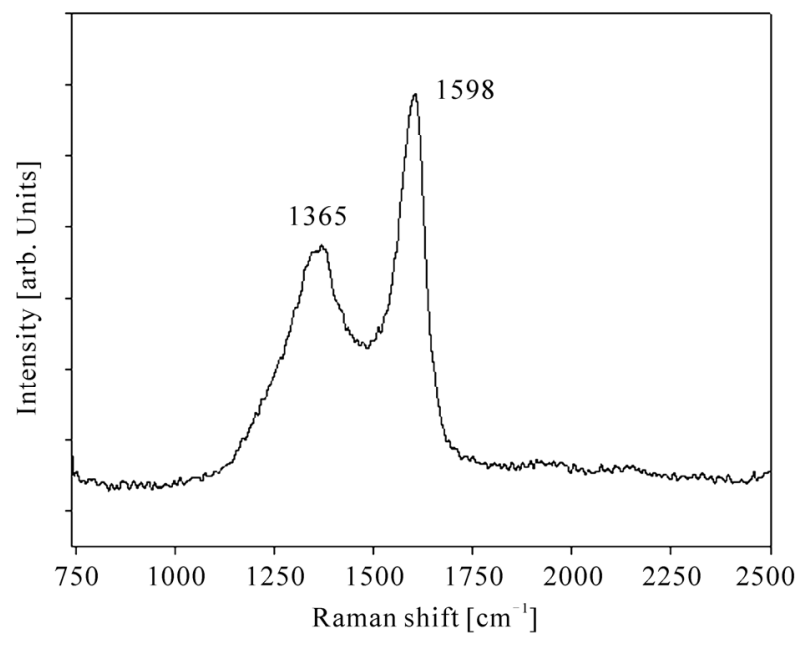

Figure 6. Raman spectrum of SWCNT-AUT SAM is shown the most characteristic features of SWCNTs (D and G band), Laser focus on SWCNT-AUT aggregated area, Laser exposure time $30 \mathrm{sec}$.

possibilities for various applications.

\section{REFERENCES}

[1] Iijima, S. (1991) Helical microtubules of graphitic carbon. Nature, 354, 56-58. doi:10.1038/354056a0

[2] Baughrman, R.H., Zakhidov, A.A. and Heer, W.A. (2002) Carbon nanotubes - the route toward applications. Science, 297, 787-792. doi:10.1038/354056a0

[3] Odom, T.W., Huang, J.L., Kim, P. and Lieber C. (1998) Atomic structure and electronic properties of single-wall ed carbon nanotubes, Nature, 391, 62-64.

[4] Hu, Y., Green M.L.H., Kim Y.H., Lee, J.Y. and Lee, C.J., Decomposition of metal carbides as an elementary step of carbon nanotube synthesis. Applied Surface Science, 26, 145-249.

[5] Vashist, S.K., Zheng, D., Al-Rubeaan, K., Luong, J.H.T. and Sheu, F.S. (2011) Advances in carbon nanotube based electrochemical sensors for bioanalytical application. Advanced Biotech, 29, 169-188. doi:10.1016/j.biotechadv.2010.10.002

[6] Ulman, A. (1990) Self-assembled organic films on gold and silver. Advanced Materials, 2, 573-582. doi:10.1002/adma.19900021203

[7] Liu, Z.F., Shen, Z.Y., Zhu, T., Hou, S.F., Ying, L.Z., Shi, Z.J. and Gu, Z.N. (2000) Chemical alignment of oxidatively shortened single-walled carbon nanotubes on silver surface. Langmuir, 16, 3569-3573. doi:10.1021/la9914110

[8] Ulman, A., Ed. (1991) An introduction to ultrathin organic films: from langmuir-blodgett to self-assembly academic, San Diego Press, San Diego.

[9] Journet, C., Maser, W.K., Bernier, P., Loisear, A., Chapell, M.L.D.L., Lefrant, S., Deniard, P., Lee, R. and Fischer, J. E. (1997) The study of the attachment of a single-walled carbon nanotube to a self-assembled monolayer using x-ray photoelectron spectroscopy. Nature, $\mathbf{3 8 8}$, 756-758. doi: $10.1038 / 41972$ 
[10] Kuzume, A., Herrero, E., Feliu, J.M., Nichols, R.J. and Schiffrin, D.J. (2002) Fullerene self-assembly and supramolecular nanostructures. Physical Chemistry, 6, 619625.

[11] Vaccarini, L., Goze, C., Aznar, R., Micholet, V., Journet, C. and Bernier, P. (1999) Mechanical purification of single-walled carbon nanotube bundles from catalytic particles. Synthetic Metals, 103, 2492-2493. doi:10.1016/S0379-6779(98)01087-X

[12] Dujardin, E., Ebbesen, T.W., Krishnan, A. and Treacy, M.M. (1998) Purification of single-walled nanotube. Journal of Advanced Materials, 10, 611-613.

[13] Protsailo, L.V. and Fawcett, W.R. (2002) Electrochemical impedance spectroscopy at alkanethiol-coated gold, in propylene carbonate. Langmuir, 18, 8933-8941. doi:10.1021/la0201218

[14] Niyogi, S., Hamon, M.A., Hu, H., Zhao, B., Bhowmik, P., Sen, R., Itkis, M.E. and Haddon, R.C. (2002) Pi-electrons in three-dimensions. Accounts of Chemical Research, 35, 1105-1113. doi:10.1021/ar010155r

[15] Huang, H., Kajiura, H., Yamada, A. and Ata, M. (2002) Electrolysis of water vapor using a fullerene-based nanotube bundles. Chemical Physics Letters, 356, 567-572. doi:10.1016/S0009-2614(02)00415-3

[16] Chapman, R.G., Lin, O.W. and Whitesides, G..M. (2002) Impact of spacers on the hybridization efficiency of mixed self-assembled dna/alkanethiol films. Langmuir, 16, 6927- 6937. doi:10.1021/la9915791

[17] Willner, I. and Riklin, A. (1994) Electrical communication between electrodes and $\operatorname{nad}(\mathrm{p})+$ dependent enzymes using pyrroloquinolinequinone-enzyme electrodes in a self-assembled monolayer configuration: design of a new class of amperometric biosensors. Analytical Chemistry, 66, 1535-1539. doi: $10.1021 / \mathrm{ac} 00081 \mathrm{a} 028$

[18] Haeussling, L., Ringsdorf, H., Schmidt, F.J. and Knoll, W. (1991) Binding of fullerene $c_{60}$ to gold surface functioalized by self-assembled monolayers of 8-amino-1-octane thiol: a structure elucidation. Langmuir, 7, 1991, 1837 1990. doi:10.1021/la00057a001

[19] Shi, Z.J., Lian, Y.F., Liao, F.H., Zhou, Z.H., Gu, Z.N., Zhang, Y.G. and Iijima, S. (1990) Cobalt ferrite aerogels by epoxide sol-gel addition: efficient catalysts for the hydrolysis of 4-nitrophenyl phosphate. Solid Sate Communicaitons, 112, 35.

[20] Janek, R.P., Fawcett, W.R. and Ulman, A. (1998) Impedance spectroscopy of self-assembled monolayers on au (111). Langmuir, 14, 3011-3018. doi:10.1021/la970980+

[21] Finklea, H.O., Snider, D.A., Fedyk, J., Sabatani, E., Gafni, Y. and Rubinstein, I. (1993) Probing pi-coupling in molecular junctions. Langmuir, 9, 3660-3667. doi:10.1021/1a00036a050

[22] Cheng, I.F., Whiteley, L.D. and Martin, C.R. (1989) U1tramicroelectrode ensembles: comparison of experimental and theoretical responses and evaluation of electroanalytical detection limits. Analytical Chemistry, 61, 762-766. doi:10.1021/ac00182a024

[23] Riklin, A. and Willner, I. (1995) Glucose and acetylcholine sensing multilayer. Analytical Chemistry, 67, 41184126. doi:10.1021/ac00118a014

[24] Widrig, C.A, Chung, C. and Porter, M.D. (1991) A catalytic 2-aminoethanethiol reduction on a gold electrode through a sulfur-gold bond formation. Journal of Electroanalytical Chemistry, 310, 335-359. doi:10.1016/0022-0728(91)85271-P

[25] Kondo, T., Sumi, T. and Uosaki, K. (2002) Electrolytic gold deposition on dodecanethiol-modified gold films, Journal of Electroanalytical Chemistry, 538, 59-63. doi:10.1016/S0022-0728(02)01000-8

[26] Byloos, M., Al-Mazna, H. and Morin, M. (1999) Formation of self-assembled. Journal of Physical Chemistry B, 103, 6554-6562. doi:10.1021/jp990642c

[27] Wong, S.S., Joselevich, E., Woolley. A.T., Cheung, C.L. and Lieber, C.M. (1998) Nanotubes as nanometer probes for chemistry and biology. Nature, 394, 52-55. doi: $10.1038 / 27873$

[28] Collins, P.G., Zettl, A., Bando, H., Thesis, A. and. Smalley, R.E. (1997) Nanotube nanodevice. Science, 278, 100-103. doi: $10.1126 /$ science. 278.5335 .100

[29] Dillon, A.C., Jones, K.M., Bekkedahl, T.A., Kiang, C.H., Bethune, D.S. and Heben, M. (1997) Storage of hydrogen in single-walled carbon nanotubes. Nature, $\mathbf{3 8 6}$, 377-379. doi: $10.1038 / 386377 \mathrm{a} 0$

[30] Kim, B.M., Qian, S. and Bau, H.H. (2005) Filling carbon nanotubes with particles. Nano Letter, 5, 873-878. doi:10.1021/n1050278v

[31] Zeng, L., Zhang, L. and Barron, A.R. (2005) Tailoring aqueous solubility of functionalized single-wall carbon nanotubes over a wide ph range through substituent chain length, Nano Letter, 5, 2001-2004. doi:10.1021/n10514994

[32] Vera, B., Anton, K., Ulrich, O., Manfred, S., Francüois, S., Robert, J. and Christophe, D. (2006) Ultrathin transparent conductive films of polymer-modified multiwalled carbon nanotubes. Journal of Physical Chemistry B, 110, 14640-14644. doi:10.1021/jp062458e

[33] Ferrari, A. and Robertson J. (2002) Interpretation of raman spectra of disordered and amorphous carbon, Physical Reviw B, 61, 14095-14107.

[34] Basca, W.S., Ugarte, D. and Chatelain A. (1994) High pressure studies of the radial breathing modes in doublewall carbon nanotubes. Physical Review B, 50, 1547315476. doi:10.1103/PhysRevB.61.14095 